\title{
On order types of systems of segments in the plane
}

\author{
Andrew Suk*
}

December 10, 2009

\begin{abstract}
Let $r(n)$ denote the largest integer such that every family $\mathcal{C}$ of $n$ pairwise disjoint segments in the plane in general position has $r(n)$ members whose order type can be represented by points. Pach and Tóth gave a construction that shows $r(n)<n^{\log 8 / \log 9}[11]$. They also stated that one can apply the Erdös-Szekeres theorem for convex sets in [10] to obtain $r(n)>\log _{16} n$. In this note, we will show that $r(n)>c n^{1 / 4}$ for some absolute constant $c$.
\end{abstract}

\section{Introduction}

We say that $n$ pairwise disjoint convex sets $\mathcal{C}$ are in general position if no three have a common tangent and for every distinct members $A, B, C \in \mathcal{C}, \operatorname{conv}(A \cup B \cup C) \neq \operatorname{conv}(A \cup B)$, that is, $C$ is not a subset of $\operatorname{conv}(A \cup B)$. We say that the ordered triple $(A, B, C) \subset \mathcal{C}$ has a clockwise (counterclockwise) orientation if there are three points $a \in A, b \in B, c \in C$ on the boundary of $\operatorname{conv}(A \cup B \cup C)$ that follow each other in clockwise (counterclockwise) order. Note that a triple $(A, B, C)$ may have both orientations. See Figure 1. Finally we say that $\mathcal{C}$ is representable by a point set $P$ if there is a bijection $f: \mathcal{C} \rightarrow P$ such that if $(A, B, C)$ has a unique orientation then $(f(A), f(B), f(C))$ has the same orientation.

Given a sequence of convex sets $\mathcal{C}$ in the plane in general position, the order type of $\mathcal{C}$ is the mapping assigning each triple $(A, B, C) \subset \mathcal{C}$ the orientation of that triple. The order type of a point set was introduced by Goodman and Pollack [6] in the early eighties, and has played a significant role in geometric transversal theory [13]. According to the conjecture of Erdős and Szekeres [7], every set of $2^{n-2}+1$ points in general position contains $n$ points in convex position. Bisztriczky and Fejes Tóth [2] generalized this conjecture as follows. Every family of $2^{n-2}+1$ disjoint convex sets in general position has $n$ members in convex position. A. Hubard and L. Montejano suggested a stronger conjecture, that every family of convex sets in general position can be represented by points. However, Pach and Tóth [11] gave a construction of $n$ pairwise disjoint segments in general position with no subfamily of size $n^{\log 8 / \log 9}$ whose order type is representable by points. They observed that it follows from a generalization of the ErdösSzekeres theorem for convex sets [10] that one can find $\log _{16} n$ members whose order type is representable by points. Our main result is:

Theorem 1. Let $r(n)$ denote the largest integer such that every family $\mathcal{C}$ of $n$ pairwise disjoint segments in the plane in general position has $r(n)$ members whose order type can be represented by points. Then there exists an absolute constant $c_{1}$ such that $c_{1} n^{1 / 4}<r(n)<n^{\log 8 / \log 9}$.

The proof of Theorem 1 is based on the following result for line transversals. Recall that a collection of convex sets in the plane $\mathcal{C}$ has a line transversal if there is a line that meets all members in $\mathcal{C}$.

Theorem 2. For any $\alpha$ such that $0<\alpha<1$, every family of $n$ convex sets $\mathcal{C}$ in the plane with no three having a common tangent line, has a subfamily $\mathcal{S} \subset \mathcal{C}$ such that either

${ }^{*}$ Courant Institute, New York. Email: suk@cims.nyu.edu 


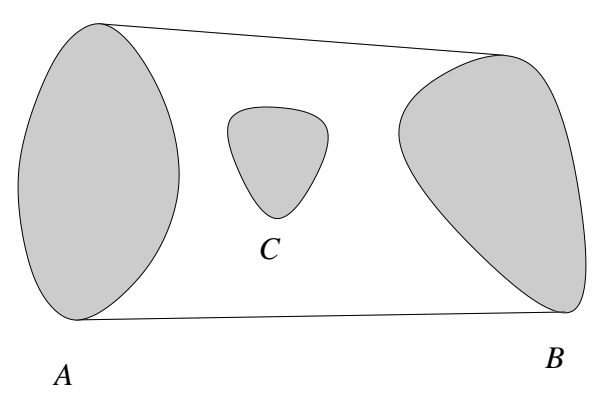

(a) $\operatorname{conv}(A, B, C)=\operatorname{conv}(A, B)$.

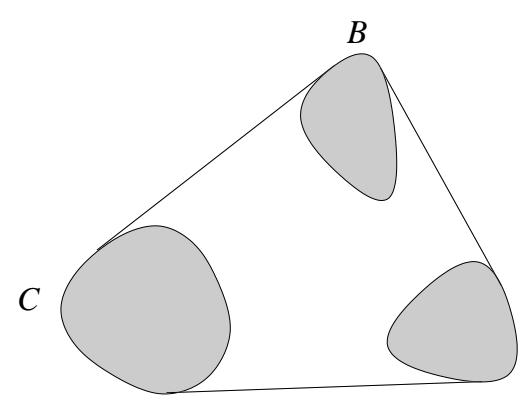

$A$

(c) $(A, B, C)$ with a counterclockwise orientation.

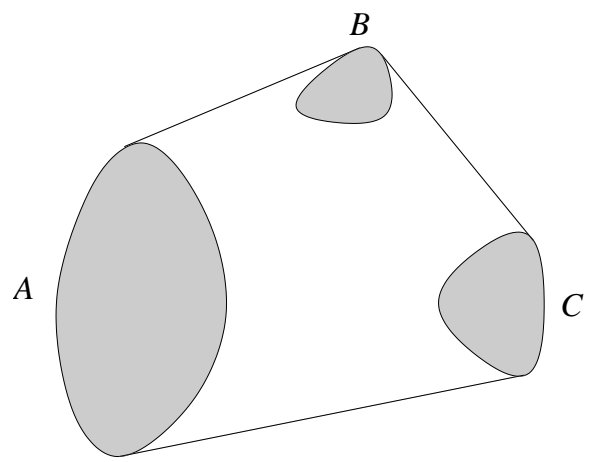

(b) $(A, B, C)$ with a clockwise orientation.

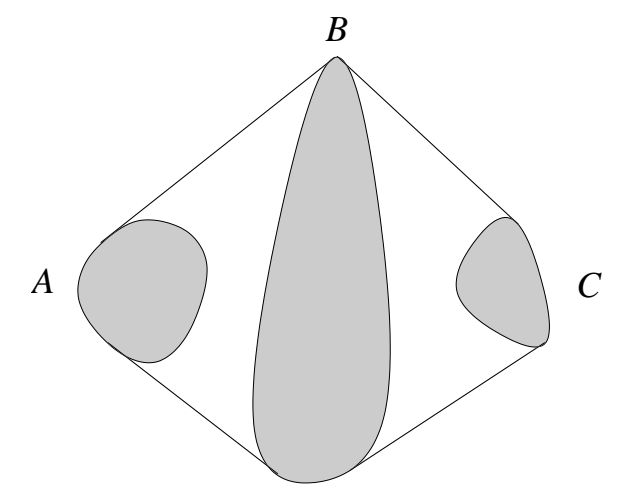

(d) $(A, B, C)$ with both a clockwise and a counterclockwise orientation.

Figure 1.

1. none of the triples in $\mathcal{S}$ have a line transversal and $|\mathcal{S}| \geq \min \left(c_{2} \alpha^{-1 / 2},(2 / 3) n\right)$

2. or $\mathcal{S}$ has a line transversal and $|\mathcal{S}| \geq c_{3} \alpha n$,

for some absolute constant $c_{2}, c_{3}$.

By setting $\alpha=n^{-2 / 3}$, we have the following corollary

Corollary 3. Every family of $n$ convex sets $\mathcal{C}$ in the plane with no three having a common tangent line, has a subfamily $\mathcal{S} \subset \mathcal{C}$ with $|\mathcal{S}| \geq c_{4} n^{1 / 3}$ such that either

1. none of the triples in $\mathcal{S}$ have a line transversal

2. or $\mathcal{S}$ has a line transversal

for some absolute constant $c_{4}$.

\section{Proof of Theorem 2}

In this section we will prove Theorem 2, which relies on two lemmas.

Lemma 4. (Spencer [12]) Let $H=(V, E)$ be an $r$-uniform hypergraph on $n$ vertices. If $|E(H)|>n / r$, then there exists a subset $S \subset V(H)$ such that $S$ is an independent set and 


$$
|S| \geq\left(1-\frac{1}{r}\right) n\left(\frac{n}{r|E(H)|}\right)^{\frac{1}{r-1}} .
$$

The second lemma is known as the fractional Helly theorem for line transversals in [9], and is due to Alon and Kalai [1]. Recall that Helly's theorem states that given a family $\mathcal{C}$ of convex sets in $\mathbb{R}^{d}$ such that every $d+1$ share a point, then all of $\mathcal{C}$ shares a point. Ever since Helly proved this beautiful theorem back in 1923 [7], there have been a vast number of Helly type results [4]. The first version of the fractional Helly theorem was proved by Katchalski and Liu [8]. We need the following.

Lemma 5. (Alon and Kalai [1]) Let $\mathcal{C}$ be $n$ convex sets in the plane such that no three share a common tangent. If there are at least $\alpha\left(\begin{array}{l}n \\ 3\end{array}\right)$ triples with a line transversal, then there exists line that intersects $\frac{\alpha}{25} n$ members in $\mathcal{C}$.

Proof of Theorem 2. Let $H$ be a 3-uniform hypergraph with $V(H)=\mathcal{C}=\left\{C_{1}, C_{2}, \ldots, C_{n}\right\}$ and $\left\{C_{i}, C_{j}, C_{k}\right\} \in$ $E(H)$ if and only if there is a line that intersects $C_{i}, C_{j}, C_{k}$. Notice that an independent set in $H$ corresponds to a subfamily of convex sets with no three having a line transversal. We can assume that $\alpha\left(\begin{array}{l}n \\ 3\end{array}\right)>n / 3$, since otherwise for large enough $n$ we can find a line that intersects at least $c_{3} \alpha n<1$ members of $\mathcal{C}$. Now the proof falls into three cases.

Case 1: If $|E(H)| \leq n / 3$, then we can get rid of all of the edges by deleting at most $n / 3$ vertices. Hence we can find an independent set of size $2 n / 3$.

Case 2: If $n / 3<|E(H)| \leq \alpha\left(\begin{array}{l}n \\ 3\end{array}\right)$, then by applying Lemma 4 above, there exists an independent set $S \subset V(H)$ such that

$$
|S| \geq \frac{2}{3} n\left(\frac{n}{3 \alpha\left(\begin{array}{l}
n \\
3
\end{array}\right)}\right)^{1 / 2} \geq c_{2} \alpha^{-1 / 2}
$$

for some absolute constant $c_{2}$.

Case 3: If $|E(H)|>\alpha\left(\begin{array}{l}n \\ 3\end{array}\right)$, then by Lemma 5 we can find a line that intersects at least $c_{3} \alpha n$ convex sets for some constant $c_{3}$.

\section{Proof of Theorem 1}

As mentioned before, the upper bound comes from a construction by Pach and Tóth [11]. For the lower bound, let $\mathcal{C}=\left\{S_{1}, S_{2}, \ldots, S_{n}\right\}$ be a collection of $n$ segments in the plane. By setting $\alpha=n^{-1 / 2}$, Theorem 2 implies that there are at least $c_{2} n^{1 / 4}$ segments such that no triple has a line transversal or $c_{3} n^{1 / 2}$ segments that can all be intersected by some line.

Case 1: If there are at least $c_{2} n^{1 / 4}$ segments $\mathcal{S} \subset \mathcal{C}$ such that every triple does not have a line transversal, then the segments "behave" like points. Hence by picking one point from each segment in $\mathcal{S}$, we have a point set that represents the order type of $\mathcal{S}$.

Case 2: Suppose there exist at least $c_{3} n^{1 / 2}$ segments $\mathcal{S} \subset \mathcal{C}$ all on a line. Without loss of generality we can assume that this line is the $y$-axis and no segment is vertical. We order the segments of $\mathcal{S}$ in the order they intersect the $y$-axis from bottom to top. By the Erdős-Szekeres theorem [7], there exists a subfamily 


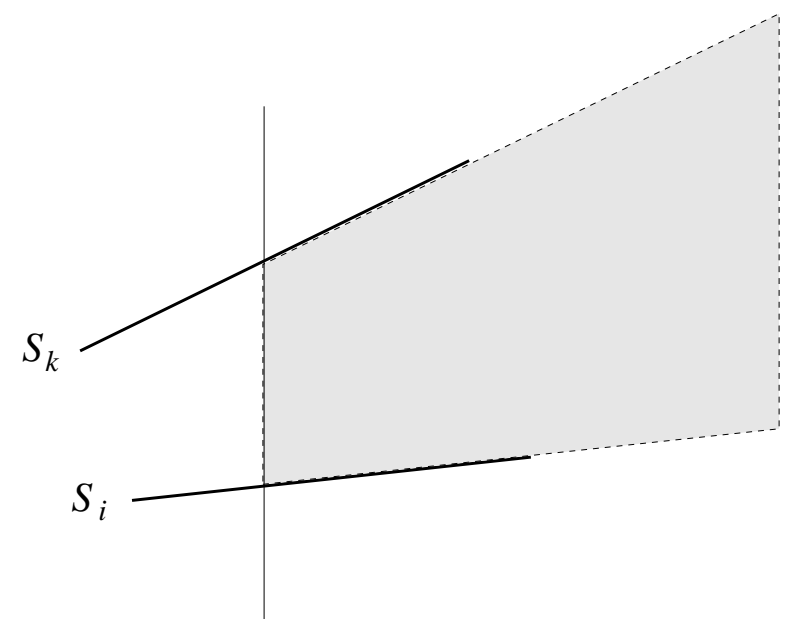

Figure 2: The region where the right endpoint of $S_{j}$ must lie.

$\mathcal{S}^{\prime} \subset \mathcal{S}$ with $\left|\mathcal{S}^{\prime}\right| \geq \sqrt{c_{2}} n^{1 / 4}$ such that the slopes of the segments are increasing or decreasing from bottom to top. With a slight abuse of notation, let us assume $\mathcal{S}^{\prime}=\left\{S_{1}, S_{2}, \ldots, S_{\left|\mathcal{S}^{\prime}\right|}\right\}$ is ordered from bottom to top and let $l_{i}, r_{i}$ denote the left and right endpoints of $S_{i}$ for each $i$. If the slopes are increasing in $\mathcal{S}^{\prime}$, then for any $S_{i}, S_{j}, S_{k}$ with $i<j<k, r_{j}$ must lie in the right half-plane below the line that contains $S_{k}$ and above the line that contains $S_{i}$. See Figure 2 .

If $\left(r_{i}, r_{j}, r_{k}\right)$ has a counterclockwise orientation, then $r_{i}, r_{j}, r_{k}$ must lie on the boundary of $\operatorname{conv}\left(S_{i} \cup\right.$ $\left.S_{j} \cup S_{k}\right)$. Therefore $\left(S_{i}, S_{j}, S_{k}\right)$ has a counterclockwise orientation (or both). Since $S_{j} \not \subset \operatorname{conv}\left(S_{i} \cup S_{k}\right)$, if $\left(r_{i}, r_{j}, r_{k}\right)$ has a clockwise orientation, then $\left(r_{i}, l_{j}, r_{k}\right)$ must lie on the boundary of $\operatorname{conv}\left(S_{i} \cup S_{j} \cup S_{k}\right)$. Hence $\left(S_{i}, S_{j}, S_{k}\right)$ has a clockwise orientation. Therefore the point set $P^{\prime}=\left\{r_{1}, \ldots, r_{\left|\mathcal{S}^{\prime}\right|}\right\}$ represents the order type of $\mathcal{S}^{\prime}$. If the slopes in $\mathcal{S}^{\prime}$ were decreasing from bottom to top, then by a similar argument, the point set $P^{\prime}=\left\{l_{1}, l_{2}, \ldots, l_{\left|\mathcal{S}^{\prime}\right|}\right\}$ would represent the order type of $\mathcal{S}^{\prime}$.

\section{Conclusion}

We would like to make two final remarks. By combining Lemma 4 and Proposition 4.1 in [1], one can easily generalize Corollary 3 for higher dimensions.

Theorem 6. Every family of $n$ convex sets in $\mathbb{R}^{d}$ with no $d+1$ have a common tangent has a subfamily $\mathcal{S} \subset \mathcal{C}$ with $|\mathcal{S}| \geq c_{d} n^{\frac{1}{d+1}}$ such that either

1. none of the $(d+1)$-tuples in $\mathcal{S}$ have a hyperplane that meets all of them,

2. or there exists a hyperplane that intersects all of $S$,

where $c_{d}$ is a constant that depends only on $d$.

Since the proof of Theorem 1 relies heavily on Theorem 2, we conjecture the following.

Conjecture 7. There exists an absolute constant $\epsilon$ such that every family of $n$ convex sets in the plane in general position has a subfamily of size $n^{\epsilon}$ whose order type can be represented by points. 


\section{References}

[1] Alon, A., Kalai, G.: Bounding the piercing number. Discrete Comput. Geom. 13, 245-256, (1995)

[2] Bisztriczky, T., Fejes Tóth, G.: A generalization of the Erdős-Szekeres convex $n$-gon theorem, Journal für die reine und angewandte Mathematik. 395 (1989)

[3] Bisztriczky, T., Fejes Tóth, G.: Convexly independent sets, Combinatorica. 10, 195-202 (1990)

[4] Eckhoff, J.: Helly, Radon, and Carathéodory Type Theorems. In: Gruber, P.M., Wills, J.M. (eds.) Handbook of Convex Geometry, pp. 389-448. North-Holland, Amsterdam, Netherlands (1993)

[5] Erdős, P., Szekeres, G.: A combinatorial problem in geometry. Compositio Math. 2, 463-470 (1935)

[6] Goodman, J. E., Pollack, R.: Multidimensional sorting, SIAM J. Comput. 12, 484-507 (1983)

[7] Helly, E.: Über Mengen konvexer Körper mit gemeinschaftlichen Punkten. Jber. Deutsch. Math. Vereinig. 32, 175-176 (1923)

[8] Katchalski, M., Liu, A.: A problem of geometry in $R^{n}$. In: Proc. Amer. Math. Soc. 75, 284-288 (1979)

[9] Matousek, J.: Lectures on Discrete Geometry. Springer-Verlag, New York (2002)

[10] Pach, J., Tóth, G.: A generalization of the Erdős-Szekeres theorem to disjoint convex sets. Discrete and Computational Geometry. 19, 437-445 (1998)

[11] Pach, J., Tóth, G.: Families of convex sets not representable by points. Indian Statistical Institute Platinum Jubilee Commemorative Volume-Architecture and Algorithms, World Scientific, Singapore, 43-53 (2009)

[12] Spencer, J.: Turán's Theorem for k-Graphs. Disc. Math. 2, 183-186 (1972)

[13] Wenger, R.: Progress in geometric transversal theory. In: Chazelle, B., Goodman, J. E., Pollack, R. (eds.) Advances in Discrete and Computational Geometry, Contemp. Math., 223, pp. 375-393. Amer. Math. Soc. (1999) 